\title{
ON LATTICE PATHS WITH DIAGONAL STEPS
}

\author{
V.K. Rohatgi
}

In [1] L. Moser and W. Zayachkowski considered lattice paths from $(0,0)$ to $(x, y)$ where the possible moves are of three types: (1) a horizontal step, (2) a vertical step, and (3) a diagonal step. They obtained an expression for the number of paths from $(0,0)$ to $(n, n)$ lying below the main diagonal except at the terminal points. In this note we extend their results to cover any point $(m, n)$ lying below the main diagonal. We use the notation of [1] and recall that

$$
\begin{aligned}
f(m, n) & =\text { total number of paths from }(0,0) \text { to }(m, n) \\
\therefore & =\sum_{r=0}^{\min (m, n)} \frac{(m+n-r) !}{(m-r) !(n-r) ! r !}
\end{aligned}
$$

and

$Q_{n}=$ number of paths from $(0,0)$ to $(n, n)$ lying below the main diagonal except at end points

$$
=\sum_{r=0}^{n-2} \frac{1}{n-r-1} \frac{(2 n-r-2) !}{(n-r) !(n-r-2) ! r !}+1 .
$$

Our method consists of counting all paths from $(0,0)$ to $(m, n)$ (with $m>n$ ) which touch or cross the main diagonal. Any path from $(0,0)$ to $(m, n)$ that touches or crosses the main diagonal must do so for the first time in any one of the following mutually exclusive ways:

(1) the path may touch or cross the main diagonal for the first time vertically (and hence from below) at the point (i, $i)$, $i=1, \ldots, n$, or

(2) the path may touch or cross the main diagonal for the first time horizontally (and hence from above) at the point (i,i) , $i=1, \ldots, n$, or

(3) the path may touch the main diagonal for the first time 
diagonally in which case it must start with a diagonal step at $(0,0)$.

It is now easy to see that the total number of paths in the first two categories is

$$
2 f(m-1, n-1)+2 \sum_{i=2}^{n} Q_{i} f(m-i, n-i)
$$

n

and that in the third is $\Sigma f(m-i, n-i)$.

$$
i=1
$$

Thus the number of paths lying below the main diagonal is given by

$$
\text { (1) } \begin{aligned}
Q_{m, n}= & f(m, n)-2 f(m-1, n-1)-2 \sum_{i=2}^{n} Q_{i} f(m-i, n-i) \\
& =\sum_{i=1}^{n} f(m-i, n-i) .
\end{aligned}
$$

The number of paths which may touch but not cross the diagonal, $Q_{m, n}^{\prime}$ is obtained by replacing $m$ by $m+1$ in (1). That is,

$$
\begin{aligned}
Q_{m, n}^{\prime}= & f(m+1, n)-2 f(m, n-1)-2 \sum_{i=2}^{n} Q_{i} f(m+1-i, n-i) \\
& =\sum_{i=1}^{n} f(m+1-i, n-i) .
\end{aligned}
$$

Finally, if $n>m$, we may ask the following question: What is the number of paths from $(0,0)$ to $(m, n)$ that cross the main diagonal vertically? The answer obviously is

$$
f(m, n)-Q_{n, m}^{\prime}
$$




\section{REFERENCE}

1. L. Moser and W. Zayachkowski, Lattice paths with diagonal steps, Scripta Mathematica, Vol. XXVI, No.3, pp. 223-229.

University of Alberta, Edmonton 